Heike Behrens (Basel)

\title{
Sprachgebrauch und Sprachbewusstsein: Implikationen der empirischen Linguistik für die Sprachtheorie
}

\section{Sprachgebrauch als Evidenz für Sprachbewusstsein?}

Die Erfassung und Analyse des Sprachgebrauchs ist durch die heute zur Verfügung stehenden Korpora und computerlinguistischen Verfahren zur linguistischen Selbstverständlichkeit geworden. Jedoch lassen sich nicht alle linguistisch und gesellschaftlich interessanten Phänomene direkt aus den Daten ableiten: Dies mag für Aspekte der Sprachstruktur, besonders dort, wo sie lexikalisch verankert ist, sehr gut funktionieren, nicht nur, um einen Zustand $\mathrm{zu}$ beschreiben, sondern auch, um Wandel und Erwerb zu dokumentieren oder ihre Mechanismen zu erklären. Diese Forschungsrichtung zielt primär auf die Ableitung und Identifikation von systematischen Erscheinungen und Prozessen in grossen Datenbanken. Jedoch bedarf es weiterer Schritte, um aus Gebrauchsdaten Aussagen über die Funktion von Sprache zu machen, insbesondere dort, wo die Sprecher die Wahl zwischen verschiedenen Optionen oder Varietäten haben, oder um zu analysieren, inwiefern der Sprachgebrauch gesellschaftliche Zustände widerspiegelt. Da jeder Sprachgebrauch letztlich auf den aktuellen Entscheidungen eines Individuums in einer bestimmten kommunikativen Situation beruht, ist weiter zu fragen, ob sich aus den Gebrauchsdaten so etwas wie das Sprachbewusstsein einer Person oder einer Gesellschaft ableiten lässt.

Aus Sicht der Systemlinguistik mögen solche Fragen eher spekulativ erscheinen, jedoch gehen sie auf eine lange Forschungstradition zurück, da die Reflexion über den „Zustand“ einer Sprache immer eine Reflexion über einen bestimmten oder einen sich verändernden Sprachgebrauch ist. Dabei kommt es in jüngster Zeit zu einer Methodenkonvergenz eher kulturanalytischer Ansätze mit Methoden der Korpuslinguistik, die verschiedene Arten von Textquellen erschliesst, um den Sprachgebrauch, seine soziolinguistischen Parameter und seinen Wandel quantitativ zu erfassen (vgl. z. B. Bürki 2013) sowie die Konzeption der historisch-geschichteten Textkorpora des 20. Jahrhunderts zu den nationalen Standards in Deutschland (www.dwds.de) und der Schweiz (schweizer-textkorpus.ch). Manifest werden overte oder koverte Annahmen über den Sprachgebrauch und das Sprachbewusstsein zudem auch bei der Auswahl der Einheiten 
und Einträge von Wörterbüchern sowie deren Annotation (Scharloth 2005; siehe auch die Beiträge von Löffler, Roth und Meier \& Hofer in diesem Band).

Die Definition von Sprachbewusstsein ist doppelgesichtig: Einerseits beschreibt es quasi wertneutral das Wissen und die Reflexion über Sprache. Sprachbewusstsein führt also $\mathrm{zu}$ differenzierten Urteilen über Sprache und idealerweise auch zu einem differenzierten und angemessenen Sprachgebrauch. Oft wird Sprachbewusstsein vermengt mit der normativ ausgerichteten Sprachpflege oder Sprachkritik. So schreiben Eichinger, Eisenberg, Klein und Storrer (2013) in ihrem Vorwort zum Sammelband Reichtum und Armut der deutschen Sprache: Erster Bericht zur Lage der deutschen Sprache, der in gemeinsamer Herausgeberschaft der Deutschen Akademie für Sprache und Dichtung sowie der Union der deutschen Akademien der Wissenschaft veröffentlicht wurde:

Das Thema „Reichtum und Armut der deutschen Sprache“ hingegen treibt eine sprachbewusste, meist sehr besorgte Öffentlichkeit zu immer erneuter Stellungnahme. Es ist geradezu erstaunlich, wie wenig die Gegenstände aus diesem Bereich in den vergangenen etwa 150 Jahren an Aktualität verloren haben. (Eichinger et al. 2013: 5)

Sprachreichtum und damit Sprachqualität wird oft mit grammatischer Komplexität und/oder gehobenem Wortschatz verglichen, und jede Abweichung davon entsprechend als ,Verfall' interpretiert. Jedoch ist gerade dieser normative Blick auf Sprache einer, der ihre Vielfalt und Dynamik auf nur wenige, typischerweise in Standardgrammatiken und -wörterbüchern kodifizierte Phänomene reduziert, und andere nicht zur Kenntnis nimmt bzw. als Nicht-Standard klassifiziert. Die empirische - Sprachwissenschaft verweigert sich seit jeher dieser Reduktion. Sie deswegen als deskriptiv, sich aus kritischen Debatten heraushaltend und ihre Verpflichtungen verletzend zu charakterisieren, ist dennoch verfehlt, geht es doch gerade darum, Reichtum und Veränderungspotenziale zu erkennen und auch zu erklären.

Ziel dieses Bandes ist es also, Forschungsrichtungen einer empirischen, gebrauchsbasierten Linguistik aufzuzeigen, die Dynamik und Wandel, Unsicherheiten über Normen und kreative Regelverletzungen als Reichtum der Sprache und als Zeichen ihrer Kreativität sowohl in der sozialen Interaktion als auch in der kognitiven Repräsentation sieht. Somit bestehen auch Implikationen für die Sprachtheorie, die sich in ihren modernen, strukturorientierten Ausprägungen oft auf Aspekte der Syntax und hier insbesondere auf die Formulierung eines schlanken Regelapparats und/oder der Identifikation der Gesetzmässigkeiten konzentriert. In den letzten Jahrzehnten gibt es eine die Vielfalt sprachlicher Erscheinungen erfassende Gegenbewegung zu dieser Engführung, die unter dem Terminus gebrauchsbasierte Linguistik (usage-based linguistics, Langacker 1987; 
vgl. Bybee 2010 für einen Überblick) die konkrete Äusserung als usage-event ins Zentrum der Sprachwissenschaft stellt. Jede Aktualisierung sprachlichen Wissens in einem solchen Sprechakt ist in einen konkreten kommunikativen Kontext eingebettet, die über die Erzeugung von grammatisch korrekten Sätzen hinausgeht:

Putting together novel expressions is something that speakers do, not grammars. It is a problem-solving activity that demands a constructive effort and occurs when linguistic convention is put to use in specific circumstances. (Langacker 1987: 65)

Die konkreten Äusserungen bilden zudem die Basis für die Abstraktion eines dynamisch gedachten sprachlichen Wissens, das zu jedem Zeitpunkt der Entwicklung aus der individuellen, akkumulierten Spracherfahrung besteht:

In usage based models of language [...] all things flow from the actual usage events in which people communicate linguistically with one another. The linguistic skills that a person possesses at any given moment in time - in the form of a structured inventory of symbolic units' - result from her accumulated experience with language across the totality of usage events in her life. (Tomasello 2000: 61-62)

Sprecher (oder Schreiber) schöpfen also in jeder Äusserung aus ihrem Wissen über die Sprache(n) in ihren mannigfaltigen Erscheinungsformen und Kontextbedingungen, und sie gestalten mit jeder Äusserung zugleich diese Sprache mit. Für die am Sprachbewusstsein orientierte Linguistik ergibt sich als Implikation die Methoden- und Datenvielfalt: Der oben zitierte Reichtum einer Sprache ergibt sich nicht nur aus den signifikanten Mustern statistik-basierter Korpusanalysen, sondern auch aus der detaillierten Beobachtung und Analyse einzelner Sprachzeugnisse. Obwohl Konventionalisierung und Frequenz wesentlich zu einem flüssigen oder gar hochgradig automatisierten Sprachgebrauch beitragen, beginnt Sprache jeweils dort, wo ein Sprachbenutzer Form und Funktion zu einem sprachlichen Symbol koppelt, es gibt kein Frequenzminimum für ,Sprache‘ (vgl. Langacker 1987, Kap. 2.1).

Dieser Sammelband beleuchtet folgerichtig das Verhältnis von Sprachgebrauch und Sprachbewusstsein aus unterschiedlichen empirischen und theoretischen Perspektiven. Im Fokus stehen qualitative korpuslinguistische Untersuchungen, die insbesondere sprachliche Phänomene jenseits der Norm (z.B. Schmidlin oder Weder) oder der lexikalischen Gebundenheit erfassen (vgl. Rothkegels Analyse des Ausdrucks von Zukunftskonzepten). Dieser Band vergegenwärtigt also einen weiten Sprachbegriff. Die gebrauchsbasierte Linguistik orientiert sich zwar an genuinen Sprachdaten, analysiert diese jedoch primär vor dem Hintergrund eines Sprachsystems: Jedoch hat ein Sprecher für jedes usage- 
event in der Regel Zugriff auf mehrere Repertoires - seien es unterschiedliche Register, Dialekte oder Sprachen, inklusive der Möglichkeit, spontan zwischen diesen Repertoires zu wechseln. Genau hier ist nicht nur die Sprachbeherrschung entscheidend, sondern das Sprachbewusstsein, das die Wahl der Option bestimmt, das aber auch reflektiert, ob diese Wahl angemessen ist. Solche Abweichungen von einem Standard werden oft als Normabweichungen klassifiziert, nicht als kreative Kompetenz, und führen so zu der Gefahr, dass Sprecher an ihrem Sprachgebrauch zweifeln. Der Entlastungsfunktion von Normen steht also die Verunsicherungsgefahr gegenüber:

Die Hilflosigkeit, mit solchen Konzepten umzugehen, zeigt sich vielerorts - in sprachpflegerischen Klagen über den Zerfall der Sprache, um Sanktionen im Sprachunterricht, in dem ein in der Regel eindimensionaler, an der Schriftlichkeit orientierter Sprachbegriff herrscht, und letztlich im Bewusstsein der Sprachbenutzer, gerade wenn sie sich selbst als an solchen Normen gescheitert oder scheiternd ansehen. (Häcki Buhofer 2014)

Dies impliziert auch, dass das Konzept der Sprachkompetenz revidiert werden muss. Mehrsprachigkeit im weitesten Sinne heisst nicht, dass Sprecher verschiedene, modular gedachte ,Systeme“ (seien es Sprachen oder Varietäten) zu einem unterschiedlichen Grad beherrschen und in der Verarbeitung getrennte Systeme interagieren, sondern dass das Gehirn ein genuin mehrsprachiges ist, das mehrsprachige Äusserungen im Sinne einer emergenten Grammatik erzeugt (vgl. die Beiträge von Filatkina, Lüdi und Franceschini).

\section{Lenkung des Sprachbewusstseins durch den Sprachgebrauch}

Das Sprachbewusstsein wird durch den Sprachgebrauch gesteuert, sowohl in der Ontogenese als auch im Erwachsenenalter, sei es aktiv im eigenen kreativen Schöpfungsakt, oder passiv durch die Beeinflussung durch gesellschaftliche Diskurse, die bestimmte Zustände als gegeben oder notwendig bzw. problematisch darstellen. Harald Burger und Natalia Filatkina stellen Sprachbiographien vor, die das Erwachen und die Verfeinerung des Sprachbewusstseins in der persönlichen Entwicklung zum Thema haben. Rosmarie Zeller und Annely Rothkegel hingegen zeigen auf, wie Bewusstseinsinhalte durch kreative Sprachkombinatorik geschaffen werden.

Der Beitrag von Harald Burger betrachtet den Spracherwerb und insbesondere das erwachende Sprachbewusstsein aus der retrospektiven Sicht der Erwachsenenliteratur. So wie Piaget versuchte, mit Hilfe von halbstrukturierten Interviews 
die Entwicklung der Begriffsbildung nachzuvollziehen, geben literarische Selbstreflexionen Aufschluss darüber, wie Kinder versuchen, die Bedeutung von abstrakten Konzepten wie Gott und Ewigkeit aus konkreten Gebrauchsbeispielen zu inferieren. Die Autorin Hilde Ziegler etwa lernt den Begriff Evakuation, als sie sich nach den Wirren des Suchens nach Schutz und des sich Verlierens der Familie wieder an die Mutter schmiegen kann. Während man in der heutigen Spracherwerbsforschung davon ausgeht, dass die Bezugspersonen in sehr hilfreicher Weise auf den Spracherwerb einwirken und sich auf den Sprachstand des Kindes einstellen, gilt bezogen auf abstrakte und z. B. gesellschaftlich oder sexuell belastete Wörter genau das Gegenteil: Wenn das Kind um Erklärung fragt, wird ihm beschieden, es sei zu klein, um es zu verstehen. Ebenso werden Fremdsprachen eingesetzt, um den Kindern etwas zu verheimlichen. Harald Burger schildert anhand vieler literarischer Quellen, welchen Reiz solche Erklärungsverweigerungen ausüben, welche Phantasien sie in Gang setzen, und welche Anstrengungen Kinder unternehmen, um diesen „verbotenen Begriffen“, die doch der Schlüssel zur geheimen Welt der Erwachsenen sind, quasi volksetymologisch auf die Spur zu kommen, und wie sie sich über das Lernen mehrerer Sprachen Zugang zu verschiedenen Welten und Diskursen verschaffen. Jenseits des Anekdotischen - der Kindermund, der unvermutete Bedeutungsbeziehungen offenlegt - zeigen die literarischen Quellen jedoch vor allem den situativen Ursprung und die situative Gebundenheit aller nicht konkreten Ausdrücke auf - seien es Abstrakta, relationale Begriffe wie Possessivpronomen oder nicht-kompositionelle Phraseologismen.

Dass Realität durch den Sprachgebrauch konstituiert werden kann, zeigt auch Rosmarie Zeller am Beispiel des Schriftstellers Christoph Geiser: Er verzichtet darauf, in seinen literarischen Werken eine fiktionale Welt zu errichten, sondern verknüpft über den sprachlichen Ausdruck Diskurse und eigentlich getrennte Welten miteinander. Geiser „macht dem Leser bewusst, dass es keine ,unschuldige‘ Sprache gibt, dass jedes Wort, jeder Ausdruck die Geschichte seines früheren Ausdrucks mitbringt“ (Zeller, in diesem Band). Geisers Sprachgebrauch löst sich von der referentiellen, denotativen Funktion der Sprache. Ihr Sinn erschliesst sich nicht ohne Interpretation vor dem Hintergrund kulturellen, gesellschaftlichen und sprachlichen Wissens. Mit diesem Wissen werden jedoch eine Vielzahl von Anspielungen und Deutungsmöglichkeiten evoziert, indem sprachliche Formen im Text verfremdet und uneigentlich verwendet werden. So lösen einfache Nebeneinanderstellungen von Wortmaterial in ihrer konzentriertesten Form eine semantische Vielschichtigkeit aus. In seinem Roman Wenn der Mann im Mond erwacht (Geiser 2008) besucht der Schweizer Protagonist einen Berliner Waschsalon der zwanziger Jahre. Durch tautologische Zusammenstellungen wie Kollektiv der Eidgenossen werden die unterschiedlichen politischen Systeme in Verbindung 
gebracht, durch Reihung von Hausgenossen und Eidgenossen die Suche nach Gemeinsamkeiten ausgelöst.

Annely Rothkegel analysiert die Genese sprachlicher Einheiten von der konzeptuellen Ebene her. So wie in den Beiträgen von Roth und Schmidlin die Formseite der sprachlichen Emergenz gezeigt wird (vgl. S. 7), kann sich auch die Semantik sprachlicher Einheiten wandeln. Das Konzept der Zukunft, wie es im Projekt „Expedition Zukunft“ der Max-Planck-Gesellschaft zum Wissenschaftsjahr 2009 gezeigt wurde (de.expedition-zukunft.org) lässt sich sowohl aus der Perspektive der technischen Entwicklung als auch aus der Perspektive des Nachhaltigkeitsdiskurses fassen. Die sich aus diesen unterschiedlichen Perspektiven ergebenden Bewertungen sind quantitativ und qualitativ nicht homolog. Im Technikdiskurs ist der Fortschritt zentral, metaphorisch werden z. B. durch technische „Assistenten“ physische oder kognitive Defizite ausgeglichen. Im Nachhaltigkeitsdiskurs ist die Perspektive der Balance insofern zentral, als künftige Entwicklungen das System nicht aus dem Gleichgewicht bringen sollen. Die Versprachlichung der Projektion des Zukünftigen lässt also Rückschlüsse auf mentale Modelle zu, die ihrerseits Weltsichten repräsentieren (Burger 1998). Ausgedrückt werden diese Projektionen einerseits durch Bedeutungserweiterungen, vor allem aber durch Kombinatorik zu (metaphorischen) Kollokationen und Phraseologismen wie in grüner Energie, die neben der Sachbezeichnung für neue technische Entwicklungen gesellschaftsspezifische positive oder negative Wertungen enkodieren und Gruppenzugehörigkeiten signalisieren.

Natalia Filatkina nimmt die von Häcki Buhofer (2002) formulierte Frage nach dem Zusammenhang von Sprachbewusstsein und Sprachgebrauch auf, indem sie die Methoden der modernen, auf die Gegenwartsprache gerichteten Attitüdenforschung (Schmidlin 2011; Cuonz 2014) auf historische Sprachzeugnisse anwendet. Sie analysiert dabei die Rolle des Fremdsprachenunterrichts, der für Kaufleute seit dem Mittelalter zum Standard der Ausbildung gehörte, insbesondere die Ausbildung im Russischen für norddeutsche Kaufmannssöhne im späten 16. und frühen 17. Jahrhundert. Unter Boris Gudunow intensivierten sich die Handelsbeziehungen, wobei in Russland der Handel traditionell auf Russisch und nicht auf Latein abgewickelt wurde. Kaufleute mussten sich also - wollten sie nicht auf Dolmetscher angewiesen sein - des Russischen in Wort und Schrift bemächtigen, und schickten zu diesem Zweck die jugendlichen Söhne nach Russland. Dokumentiert sind diese Sprachbeziehungen in einigen Selbstzeugnissen der Betroffenen sowie in Sprachlehrbüchern aus der Zeit. Filatkina argumentiert, dass diese Quellen überregional standardisierte Muster des humanistischen lexikographischen und sprachdidaktischen Diskurses repräsentieren, so dass sich aus diesen Dialogen kollektive und exemplarische Modelle für bestimmte Sprechhandlungen, z. B. Verkaufsgespräche, ableiten lassen. Bei diesen 
Quellen handelt es sich nicht um einen primär präskriptiven und normativen Zugang zur Sprache, sondern sowohl in den Selbstzeugnissen als auch in den Lehrwerken um gesprochene Sprache in alltäglichen Kommunikationssituationen, die über die sprachlichen Formen hinaus implizit pragmatisches und kulturelles Wissen vermitteln und so zu dem „bewussten“ und sensiblen Sprachgebrauch der Kaufleute beitragen, der für erfolgreiche Handelsabschlüssen nötig ist.

\section{Die Konstitution von Sprache in der Lexikographie und Wahrnehmungsdialektologie}

Während es aus Sicht der Sprachkontakts- und Mehrsprachigkeitsforschung problematisch ist, von einer strikten Trennung sprachlicher Systeme auszugehen (siehe unten, Kap. 4), ist Sprache dennoch ein wesentliches Element der eigenen Identität, mit dem sich Sprecher von anderen Gruppen absetzen. Die Bestimmung der Kriterien, nach denen Elemente Eingang in ein standardsprachliches oder regionales Wörterbuch finden, ist seit jeher eine der wesentlichen Fragestellungen der Lexikographie. Heinrich Löffler nähert sich diesem Thema aus der idiosynkratischen Sicht eines der ersten Wörterbuchschreibers, während Stefanie Meier und Lorenz Hofer eine moderne Variante des crowdsourcing durch Online-Befragung in der entsprechenden Sprachgemeinschaft vorstellen.

Heinrich Löffler verortet den Beginn der Reflexionen zu Sprachbewusstsein und Sprachgebrauch in die frühe Neuzeit und Reformation, als man sich um die Emanzipation der Volkssprachen bemühte, aufbauend auf der Etablierung von Handelssprachen seit dem frühen Mittelalter (vgl. auch Filatkina in diesem Band). Diese Bewegung fand ihren Höhepunkt in den Sprachgesellschaften des 17. bis 19. Jahrhunderts. Der Basler Johann Jakob Spreng (1699-1768) gehörte in diesen Kreis. Er hat, neben einem 20-bändigen, auf intensiven Quellenstudien beruhenden und bislang unveröffentlichten deutschen Wörterbuch, mit seinem zwischen 1740 und 1768 entstandenen Idioticon Rauracum (Löffler 2014) die Tradition der Regionalwörterbücher mitgegründet. Wie bei den Grosswörterbüchern zeigt sich auch hier die bewusstseinsbildende Kraft der Einzelautoren: Spreng entschied sich für einen Zugang, der die typischen Besonderheiten des Baseldeutschen hervorhob, aber auch seine keltischen Wurzeln betonte. So spiegelt dieses Wörterbuch einerseits die Lebenswirklichkeit der Sprachbenutzer zu der Zeit, wie sie Spreng wahrgenommen hat, und positioniert zugleich das Baseldeutsche in der Landschaft der Hoch- und Bildungssprachen. 
Derartig idiosynkratische introspektive Zugänge zur Lexikographie, verbunden mit zeittypischen pädagogisch-politischen Motiven, entsprechen nicht mehr dem heutigen methodischen Standard. Wörterbücher werden ,objektiviert', indem sie z. B. durch (institutionell legitimierte) Autorengruppen herausgegeben werden und in der Regel eine korpuslinguistische Basis haben, die den tatsächlichen Sprachgebrauch als ,Sprachwirklichkeit“ widerspiegeln und subjektiven Entscheidungen vorbeugen soll (Klosa 2007; Klein 2004). Für Dialektwörterbücher ergibt sich jedoch das Problem, dass es kaum Korpora einerseits jenseits der Mundartliteratur gibt, und es andererseits eine hohe Erwartungshaltung der Rezipienten gibt, dass diese Wörterbücher das repräsentieren sollen, was sie selbst als typisch und kennzeichnend bzw. als Teil der lokalen Tradition auffassen. Stefanie Meier und Lorenz Hofer charakterisieren das in diesem Spannungsfeld zur Verfügung stehende lexikographische Methodenrepertoire, dem das Neue Baseldeutsch Wörterbuch (Gasser, Häcki Buhofer \& Hofer 2010) die Online-Befragung hinzufügt. Die Teilnahme von fast 4000 Personen zeigt das grosse Interesse der Bevölkerung an der Mundart als Teil der persönlichen und regionalen Identität. Die Probanden beurteilten die Wörter nicht nur danach, ob sie ihnen bekannt und/oder Teil ihres eigenen Wortschatzes waren, sondern sie lieferten auch Beispielsätze dafür, wie sie dieses Wort verwenden würden, was es bedeutet und welche ähnlichen Wörter es gibt. Die Online-Befragung erlaubte es, sowohl Bedeutungsvarianten als auch nicht mehr gebräuchliche, aber von den Sprachbenutzern als zum Dialekt gehörende Wörter zu identifizieren.

Auch die Wahrnehmungsdialektologie beschäftigt sich mit den durch die Sprachbenutzer empfundenen Grenzen und Einheiten. Helen Christen diskutiert in ihrem Beitrag ein sprecherbasiertes Modell der Dialektologie. Traditionellerweise werden Dialekte auf Basis von Isoglossenbündeln eingeteilt, die auf konkreten sprachlichen Merkmalen beruhen, etwa dem Vollzug oder Nicht-Vollzug bestimmter Lautverschiebungen, oder lexikalischer bzw. morphosyntaktischer Merkmale. Diese werden oft mit siedlungsgeschichtlichen Befunden in Übereinstimmung gebracht. Jedoch definieren Sprecher Dialekte eher auf Basis ihres Zugehörigkeitsgefühls, etwa ,Kantonsdialekte‘, die es auf Basis traditioneller dialektologischer Merkmale eigentlich nicht gibt. Das Zugerdeutsch etwa bezeichnet die Sprechweise im Kanton Zug, ist jedoch eher das Resultat einer ,Innenausrichtung‘ als eine objektivierbare sprachliche Grösse, denn die entsprechenden Isoglossen verlaufen nicht isomorph. Mit Hilfe moderner dialektometrischer Methoden lassen sich die Beziehungen zu den Nachbardialekten genauer fassen: Auf der Basis von 216 Merkmalen gibt es in der Tat weder ein eindeutiges Zentrum des Dialekts noch eindeutige Kennzeichen, sondern Merkmalsbündel. Dennoch zeigen sich die stärksten Unterschiede an den Grenzen zu den anderen grossen Dialektzentren Zürich und Luzern, während die Unter- 
schiede zum südlich gelegenen Kanton Schwyz weniger stark ausgeprägt sind. Wie verhalten sich diese Befunde nun wahrnehmungsdialektologisch? Sprecherurteile bestätigen, dass der Kanton Zug einen Mischdialekt habe, die Abgrenzung von Zürich und Luzern aber schwierig sei. So ergibt sich die Abgrenzung dieses Dialekts gerade aus seiner Zwischenstellung. Die Haltung der Sprecher zu ihrem Dialekt ist ambivalent: Einerseits wird anerkannt, dass es kein ,richtiger‘ Dialekt sei, andererseits wird beklagt, dass das Zugerdeutsche im Zuge steigender Mobilität an Präsenz verliere. Diese Ambivalenz lässt sich auflösen, wenn man davon ausgeht, dass einige Sprecher aus der Nahperspektive eine sehr genaue Wahrnehmung von subtilen Dialektmerkmalen und -grenzen auch innerhalb eines Dialektgebiets haben.

\section{Die Emergenz sprachlicher Einheiten im Sprachgebrauch}

Wenn der Sprachgebrauch eine hohe Sensibilisierung in der Sprachwahrnehmung bewirkt, ist es nur folgerichtig, dass die Kontexte des Sprachwandels am Sprachgebrauch ablesbar sind. Tobias Roth untersucht, unter welchen Bedingungen Sprecher zwischen Konstruktionen wählen, während Regula Schmidlin und Mirjam Weder die Emergenz von Strukturen aus bestehenden oder sich entwickelnden neuen sprachlichen Mustern belegen. Peter Ďurčo macht sich die quantitativen und qualitativen Methoden der Korpuslinguistik zunutze, um möglichst ,musterhafte، Materialien für Zweitsprachlerner zu entwickeln.

Ein zentrales Thema dieses Bandes ist, dass Sprecher Optionen im Sprachgebrauch haben. Tobias Roth wendet korpuslinguistische Methoden an, um zu analysieren, unter welchen Bedingungen Sprecher Komposita (Grüntee) oder Kollokationen (grüner Tee) verwenden. Erfasst werden sowohl Frequenzmasse, Assoziationsmasse als auch funktionale Analogien (mit Hilfe der latent semantic analysis, LSA). Die Entscheidungen scheinen weniger durch allgemeine Faktoren der Sprachökonomie wie Kürze oder Frequenz bedingt zu sein, als vielmehr durch den syntaktischen Kontext, in dem beispielsweise gerade in längeren und komplexeren Texten eine Tendenz zur Verdichtung durch Komposita festzustellen ist.

Regula Schmidlin beschäftigt sich mit durch Jugendsprache ausgelöstem Sprachwandel. Sie verwendet einen dynamischen Sprachbegriff, der sich Haspelmaths These anschliesst, dass Grammatik das „Nebenprodukt des Sprechens in sozialer Interaktion“ sei (Haspelmath 2002: 10) und Jugendliche diejenigen seien, die durch kreativen Gebrauch sprachlicher Mittel zugleich auffallen und sich von der Norm abgrenzen wollen. Seit Androutsopoulos (1998) wendet sich das 
Interesse verstärkt dem Einfluss der Jugendsprache auf die Grammatik zu, der sich vor allem in Phänomenen der gesprochenen Sprache manifestiert, die von Standardgrammatiken nicht erfasst werden. Theoretisch lehnt sich diese Forschung an die Grammatikalisierungsforschung an. Schmidlin zeigt anhand der NexI-Konstruktion (voll + NP wie in voll die Überraschung) an, wie diese der Intensivierung dienende Konstruktion sich in Analogie zu verschiedenen anderen Intensivierungs-Konstruktionen entwickelt hat und eine Lücke im System füllt, die ihrerseits wieder Raum öffnet für Analogiebildungen (mega NP). NexI steht dabei für nominalphrasen-externe Intensivierer, die anderen syntaktischen Bedingungen folgen als die etablierten Adverbien wie sehr oder viel. Schmidlin belegt, wie die NexI-Konstruktion von Jugendlichen grammatikalisiert wurde und eine Lücke im Steigerungsparadigma des Deutschen füllt. Das Sprachbewusstsein in Form des Bemerkens einer fehlenden Ausdrucksmöglichkeit treibt also auch hier den innovativen Sprachgebrauch an und damit die Verfestigung der neuen Struktur im Sprachsystem durch Grammatikalisierungsprozesse.

Mirjam Weder befasst sich mit Normierungsprozessen auf der Textebene. Am Beispiel fachwissenschaftlicher Texte aus mehreren Disziplinen in den Geisteswissenschaften, in denen es wegen der vielfältigen Genres und Forschungstraditionen keine normative Struktur von Artikeln wie z.B. in den Natur- und empirischen Sozialwissenschaften gibt, untersucht sie den Textteil ,Einleitung ' in Hinblick auf seine konzeptuelle Struktur, die sogenannten moves, und deren Verbalisierung durch bestimmte Phrasen und Textroutinen. Motiviert ist ihre Fragestellung durch die praktische Erfahrung, dass Studierende dieser Disziplinen in Ermangelung normativer Texttraditionen oft grosse Schwierigkeiten haben, ihre wissenschaftlichen Arbeiten zu strukturieren. Es zeigt sich, dass die Disziplinen unterschiedlich stark durch Textmuster geprägt sind, dass jedoch einige pragmatische Funktionen wie Hypothesen oder Fragestellungen formulieren kaum verbalisiert werden. D.h., dass viele der untersuchten wissenschaftlichen Texte nur bedingt geeignet sind, durch ihre strukturelle und lexikalisierte Musterhaftigkeit metadiskursives Wissen bei den Studierenden hervorzurufen, wie es bei Artikeln, die ihre Arbeitsschritte offenlegen, der Fall ist.

Die soeben diskutierten emergenten Strukturen müssen in der Lexikographie und Grammatikschreibung adäquat erfasst werden, beziehen sich jedoch auf ein einzelnes Sprachsystem (auch wenn dies durch Sprachkontakt beeinflusst werden kann).Während sich hier je Kriterien finden lassen, die zur Bestimmung relevanter Einheiten führen, wird dieses Problem komplex, wenn es darum geht, Phänomene $\mathrm{zu}$ erfassen, die Systemgrenzen überschreiten. Im Falle von Lehrwerken und Lernerwörterbüchern kann eine empirisch abgesicherte, frequenzbasierte Auswertung des zielsprachlichen Standards helfen, um Lehrmaterial zu entwickeln, wie Peter Ďurčo vorschlägt. Er schliesst an die Methode Roths (Roth 2014) an, 
indem er sie für mehrsprachige Kollokationenwörterbücher funktional machen will. Traditionelle Wörterbücher enthalten kaum Kollokationen, obwohl gerade sie durch ihre Unvorhersagbarkeit massive Lernprobleme im Fremdsprachenerwerb verursachen, und ein unidiomatischer, oft durch die Erstsprache beeinflusster Gebrauch von Kollokationen selbst bei sehr weit fortgeschrittenen Lernern zu beobachten ist. Empirisch fundierte mehrsprachige Wörterbücher sollen also die für Lerner wichtigsten und häufigsten Kollokationen erfassen und die Benutzer für strukturelle und semantische Besonderheiten sensibilisieren.

\section{Die Auflösung von Systemgrenzen in der Mehrsprachigkeit}

Einem anderen Aspekt der Emergenz von Struktur widmen sich Csaba Földes, Georges Lüdi und Rita Franceschini: In mehrsprachigen Kontexten kommt es zu gemischtsprachlichen Strukturen, die durchaus intentional sind, um - wie bei Dialekten - identitätsmarkierend $\mathrm{zu}$ wirken, und/oder aus den reichen geteilten sprachlichen Ressourcen zu schöpfen.

Csaba Földes untersucht kontaktsprachliche Phänomene in der Lokalpresse ungarndeutscher Gebiete, in denen neben Ungarisch und Hochdeutsch ungarndeutsche Ortsmundarten gesprochen werden. In der Lokalpresse lassen sich Inferenzen auf allen sprachlichen Ebenen beobachten, insgesamt ergibt sich der Eindruck einer vergleichsweise geringeren Verwendung von Phraseologismen sowie ein stärker an der Mündlichkeit orientierter Sprachstil als in den ungarischen oder deutschen nationalsprachlichen Presseerzeugnissen, die in diesem Gebiet ebenfalls gelesen werden. Besonders aufschlussreich sind ,Kontaktphraseologismen' und kreative Metaphern, in denen transkulturelle Konzeptualisierungen nachweisbar sind, etwa eine Pleite erleiden analog zum Ungarischen ,ein Versagen erleiden‘. Für Földes sind solche Schwankungen und Eklektizismen im Sprachgebrauch Ungarndeutscher kein Beleg für sprachliche Defizite in der Standardsprache, sondern ein „sprachlicher Mikrokosmos“, der quasi als „dritter Raum“ die strukturellen, konzeptuellen und kulturellen Transfer- und Kontaktmöglichkeiten in dieser mehrsprachigen Sprachgemeinschaft beinhaltet.

Wie Földes wendet Georges Lüdi sich gegen ein Konzept von Mehrsprachigkeit, das den perfekten bilingualen Sprecher zur Norm erhebt. Die Konzentration auf solche Ausnahmeerscheinungen verstellt den Blick auf die wahre Bedeutung von Mehrsprachigkeit, weil sich hier die Idee eines am normativen Ideal orientierten präskriptiven und elitären Sprachgebrauchs manifestiert, der die weniger perfekte Sprachbeherrschung als defizitär gelten lässt. Mit Cook (1991, 1992) 
wendet er sich gegen die Idee einer additiven Mehrsprachigkeit, in der neue Sprachen zu der perfekt beherrschten Erstsprache hinzukommen, hin zu einem Konzept der Multikompetenz, das Mehrsprachige als in mehreren Sprachen Begabte sieht, ohne dass eine der Sprachen notwendigerweise perfekt beherrscht wird oder Perfektion das Ziel ist. In dieser Konzeption entsteht das - mehrsprachige - Sprachsystem aus den Sprachhandlungen der Benutzer: von langue zu languaging (Thorne \& Lantolf 1987). Im Zuge dieses Richtungswandels zu dynamischen, emergenten und interaktionsbasierten Auffassungen von Sprache entwickelten sich Theorien, die syntaktische Strukturen als veränderliches Sediment von sprachlichen Interaktionen sehen (etwa das Konzept der Emergent Grammar, Hopper 1987) und die multimodale Signale als Teil des Sprachsystems auffassen (z. B. Mondada 2001). Diese Konzepte erlauben es, auch mehrsprachige Syntaxen $\mathrm{zu}$ erfassen, code-switching also nicht als ,Produktionsmissgeschick oder Abweichung vom monolingualen Standard $\mathrm{zu}$ sehen, sondern als intentionale Sprachproduktion, weil Sprecher zwischen einsprachigem oder mehrsprachigem Modus wählen können. Die Akzeptanz einer solchen Konzeption von emergenter mehrsprachiger Grammatik hat vielfältige Implikationen für unser Konzept von sprachlichen Normen und den Schulunterricht als Vermittler und Sanktionierer einer solchen Normativität.

Auch Rita Franceschini nimmt Vivian Cooks Überlegungen zur Multikompentenz (Cook 1991, 1992) als Ausgangspunkt für ihre Überlegungen über eine adäquate Theorie der Sprache und ihrer mentalen Repräsentation. Am Beispiel von Sprachbiographien und neurolinguistischen Versuchen zeigt sie, dass eine system- und normorientierte Sicht auf Sprache bei Mehrsprachigen zu kurz greift. Zum einen werden hier oft inzidentelle Sprachkenntnisse übersehen, weil die Sprachkompetenzen meist in der Muttersprache bzw. in Schul- oder Landessprachen gemessen werden. Die meisten Menschen verfügen aber über weit mehr Sprachkenntnisse, die sie variabel und kompetent im Sinne einer kommunikativen Funktionalität einsetzen. Neben den ,Brocken' der Sprache eines Urlaubslandes lassen sich hier auch mehr oder weniger rudimentäre Kenntnisse von Sprachen von Freunden oder Bekannten denken, sowie insbesondere auch die diversen Lekte der eigenen Sprache. In Sprachbiographien von ladinischsprachigen Kindern in Südtirol zeigte sich, dass diese Sprachkenntnisse und die damit verbundenen Situationen oft als subjektiv wichtiger empfunden werden als die im Schulunterricht vermittelten Sprachen. Die grosse subjektive Bedeutung dieses unfokussierten, quasi nebenbei verlaufenden Spracherwerbs ist der Relevanz der sozialen Funktion von Sprache geschuldet (vgl. Franceschini 1999, 2012). Das inzidentelle Lernen findet in für Sprecher wichtigen und erinnerungswürdigen Situationen statt. Solch implizites Lernen führt somit auch zu kognitiven Effekten. Rita Franceschini diskutiert neurolinguistische Experimente, die zeigen, dass 
overte und koverte mehrsprachige Probanden in einer stillen Erzählaufgabe (silent free naration task; vgl. Kim, Relkin, Lee \& Hirsch 1997) gleich gut abschnitten, unabhängig davon, ob die zweite oder dritte Sprache aktiv verwendet wurde oder nur eine oft gehörte Umgebungssprache war.

\section{Implikationen für die Sprachtheorie}

Insgesamt zeigt dieser Band, dass eine dynamische Konzeption von Sprache, die den kulturellen Kontexten des Sprachgebrauchs und den individuellen Quellen des Sprachbewusstseins Rechnung trägt, Beziehungen zwischen verschiedenen Wissenschaftstraditionen und Forschungsfragen herstellen kann: Die von Rosmarie Zeller beschriebenen Mechanismen zur Herstellung fiktiver Welten in der Literatur finden sich ebenso in den von Annely Rothkegel analysierten Zukunftsdiskursen der Technik, in denen durch kreative Wortkombinatorik positiv oder negativ konnotierte neue Welten projiziert werden. Das Problem der Abgrenzung sprachlicher Systeme zeigt sich nicht nur in der individuellen Sprachwahl, der Schaffung von Zugehörigkeiten und Identitäten durch das Lernen verschiedener Varietäten, wie sie in der Dialektologie (Christen), der Sprachkontaktforschung (Földes), aber auch in der ontogenetischen Perspektive des Spracherwerbs und der Sprachbiographie (Burger, Filatkina, Lüdi, Franceschini) thematisiert wurden, sondern auch in den methodischen Überlegungen, die der Kodifizierung solcher Zustände in Wörterbüchern zugrunde liegen (Löffler, Meier und Hofer). Ebenso wurde aufgezeigt, dass der Systemcharakter der Sprache eher als musterhaft denn als kategorisch definiert zu sehen ist: Tobias Roth, Regula Schmidlin und Mirjam Weder charakterisieren Phänomene auf Wort-, Satz- und Textebene jenseits etablierter Kategorien und Normen, die jedoch im Zuge ihrer wachsenden Frequenz und/oder Akzeptanz Muster für analoge Bildungen schaffen und Spielraum für alternative Konstruktionen bieten. Ob das Konzept abgrenzbarer Sprachsysteme linguistisch, sozial und kognitiv überhaupt haltbar ist, stellen die Beiträge zur Mehrsprachigkeit (Ďurčo, Földes, Lüdi, Franceschini) in Frage, indem sie Phänomene diskutieren, die mit diesem Konzept nur unzureichend erfasst werden können.

Eine Sprachtheorie, die den Reichtum der Sprache und der Sprecher abbildet, sollte nicht nur auf die Analyse und Bestimmung des System fokussiert sein, sondern die Analogien zwischen den diese Phänomene hervorbringenden kulturellen, sozialen und kognitiven Prozessen aufzeigen. Ein solcher prozessorientierter Blick kann Gemeinsamkeiten, wenn nicht gar Regelhaftigkeiten zwischen unterschiedlichen Phänomen- und Forschungsbereichen in der Linguistik aufzeigen (Behrens \& Pfänder 2014; Beckner et al. 2009). 


\section{Danksagung}

Für die Konzeption und Umsetzung dieses Sammelbandes danken wir vielen: Die Philosophisch-Historische Fakultät der Universität Basel und der Hochschulrat der Universität Freiburg haben uns mit Beiträgen an den Druckkostenzuschuss unterstützt.

Lorenz Hofer hat bei der Planung und Konzeption dieses Sammelbandes mitgewirkt und entscheidende Impulse geliefert. Daniel Gietz vom de Gruyter Verlag hat die Entstehung dieses Bandes konstruktiv begleitet. Nadine Mathys (Universität Freiburg) hat alle Beiträge redigiert; Steffen Siebenhüner, Julia Voegelin und Catherine Diederich (Universität Basel) sowie Alexandra Schiesser (Universität Freiburg) haben zur Endredaktion beigetragen.

Wir widmen diesen Band unserer Kollegin Annelies Häcki Buhofer, die uns durch ihre wissenschaftliche Neugier und den Mut, neue Pfade zu gehen und unkonventionell zu denken, eine Quelle der Inspiration ist.

\section{Literatur}

Androutsopoulos, Jannis K. (1998): Deutsche Jugendsprache. Untersuchungen zu ihren Strukturen und Funktionen. Frankfurt am Main: Peter Lang.

Behrens, Heike \& Stefan Pfänder (2013): Die Entstehung von Sprache. In Peter Auer (Hrsg.), Sprachwissenschaft: Grammatik - Interaktion - Kognition. Stuttgart, Weimar: Metzler, 319-346.

Beckner, Clay, Richard Blythe, Joan Bybee, Morten H. Christiansen, William Croft, Nick C. Ellis, John Holland, Jinyun Ke, Diane Larsen-Freeman \& Tom Schoenemann (2009): Language is a complex adaptive system: Position paper. Language Learning 59(Supplement 1), 1-27.

Bybee, Joan L. (2010): Language, Usage and Cognition. Cambridge: Cambridge University Press.

Bürki, Andreas (2013): Formulaic Language and its Cultural Context: A Diachonic Perspective. Dissertation, Universität Basel.

Cook, Vivian J. (1991): The poverty-of-the-stimulus argument and multicompetence. Second Language Research, 7, 103-117.

Cook, Vivian J. (1992): Evidence for multicompetence. Language Learning 42, 557-591.

Cuonz, Christina (2014): Sprachliche Werturteile von Laien. Eine sozio-kognitive Analyse. Tübingen: Francke.

Gasser, Markus, Annelies Häcki Buhofer \& Lorenz Hofer (2010): Neues Baseldeutsch Wörterbuch. Basel: Christoph Merian.

Eichinger, Ludwig M., Peter Eisenberg, Wolfgang Klein \& Angelika Storrer (2013): Vorwort - Die deutsche Sprache ist, wie sie ist. In: Deutsche Akademie für Sprache und Dichtung \& Union der deutschen Akademien der Wissenschaften (Hrsg.): Reichtum und Armut der deutschen Sprache. Berlin: de Gruyter, 5-13. 
Geiser, Christoph (2008): Wenn der Mann im Mond erwacht. Ein Regelverstoss. Zürich: Amman.

Franceschini, Rita (1999): Italiano di contatto. Parlanti occasionali e riattivazioni di conoscenze non focalizzate. Habilitationsschrift, Universität Basel.

Franceschini, Rita (2012): Unfokussierter Spracherwerb in Kontaktsituationen. Sprachexposition als Teil des Sprachwandels. Sociolinguistica 26, 41-57.

Häcki Buhofer, Annelies (2014): ,Unachtsam‘ und ,rigoros unsicher - zwei Facetten der Wertung beim Spracherwerb in der diglossischen Norm. Plenarvortrag auf dem Symposion Deutschdidaktik. FHNW Basel, 7.9.2014.

Häcki Buhofer, Annelies (2002): Steuert Sprachbewusstheit den eigenen Sprachgebrauch? Überlegungen zum Zusammenhang an Beispielen aus der deutschen Schweiz. Der Deutschunterricht 3, 18-30.

Haspelmath, Martin (2002): Grammatikalisierung: Von der Performanz zur Kompetenz ohne angeborene Grammatik. In: Sibylle Krämer \& Ekkehard König (Hrsg.): Gibt es eine Sprache hinter dem Sprechen? Frankfurt am Main: Suhrkamp, 262-286.

Hopper, Paul (1987): Emergent grammar. Berkeley Linguistic Society 13, 139-157.

Kim, Karl H. S., Norman R. Relkin, Kyoung-Min Lee \& Joy Hirsch (1997): Distinct cortical areas associated with native and second languages. Nature 388, 171-174.

Klein, Wolfgang (2004): Vom Wörterbuch zum Digitalen Lexikalischen System. Zeitschrift für Literaturwissenschaft und Linguistik 136, 10-55.

Klosa, Anette (2007): Korpusgestützte Lexikographie: besser, schneller, umfangreicher? In: Kallmeyer, Werner \& Gisela Zifonun (Hrsg.): Sprachkorpora - Datenmengen und Erkenntnisfortschritt. Berlin, New York: de Gruyter, 105-122.

Langacker, Ronald W. (1987): Foundations of Cognitive Grammar. Vol. 1: Theoretical Prerequisites. Stanford: Stanford University Press.

Löffler, Heinrich (Hrsg.) (2014): Idioticon Rauracum. Johann Jakob Spreng. Baseldeutsches Wörterbuch. Basel: Schwabe.

Mondada, Lorenza (2001): Pour une linguistique interactionnelle. Marges Linguistiques 1, 142162.

Scharloth, Joachim (2005): Sprachnormen und Mentalitäten: Sprachbewusstseinsgeschichte in Deutschland im Zeitraum von 1766 bis 1785. Tübingen: Niemeyer.

Schmidlin, Regula (2011): Die Vielfalt des Deutschen: Standard und Variation. Gebrauch, Einschätzung und Kodifizierung einer plurizentrischen Sprache. Berlin, New York: de Gruyter.

Roth, Tobias (2014): Wortverbindungen und Verbindungen von Wörtern. Tübingen: Francke.

Thorne, Steven L. \& James P. Lantolf (2007): A linguistics of communicative activity. In: Sinfree Makoni \& Alastair Pennycook (Hrsg.): Disinventing and Reconstituting Languages.

Clevedon: Multilingual Matters, 170-195.

Tomasello, Michael (2000): First steps toward a usage-based theory of language acquisition. Cognitive Linguistics 11, 61-82. 


\section{Sprachgebrauch und Sprachbewusstsein}

Implikationen für die Sprachtheorie

Herausgegeben von

Regula Schmidlin, Heike Behrens und Hans Bickel 